\title{
Correlación de factores subjetivos y objetivos incidentes en el rendimiento académico de la carrera de Banca y Finanzas
}

\author{
MSc. José Daniel Guido Chávez \\ Docente investigador \\ UNAN-Managua, FAREM-Carazo \\ josielguivez@hotmail.com
}

DOI: http://dx.doi.org/10.5377/torreon.v6i16.6556

Palabras claves: Rendimiento Académico, Factores subjetivos y objetivos, $V$ de Cramer, Tau-C de Kendall, Phi

\section{RESUMEN}

on el propósito de correlacionar los factores subjetivos y objetivos que inciden en el rendimiento académico de los estudiantes en la carrera Banca y Finanzas, de la Facultad Regional Multidisciplinaria de Carazo (FAREM - Carazo) de la UNAN Managua, en el período 2011-2015, se presenta un estudio de tipo observacional, correlacional y analítico, que tradicionalmente es analizado solamente como un estudio de tipo descriptivo. Mediante la aplicación de las pruebas estadísticas no paramétricas $V$ de $C$ ramer, Tau- $C$ de Kendall y Phi se facilita la evolución del estudio, considerando factor primordial, la naturaleza de las variables para poseer una estructura estadística bien definida; además tener premisas claras para construir la correlación o asociación entre las variables, estas premisas deben ser redactadas en forma de hipótesis, las cuales guían el propósito del estudio.

\section{INTRODUCCIÓN}

Los diversos teóricos que han estudiado el rendimiento académico, han descrito, desde diversas perspectivas, enfoques, estados (social, económico, cultural, político, educativo) de los actores directos, han consensuado que su significación es multicausal o multifactorial, abarcando todas las dimensiones estudiadas; así que, para realizar una evaluación del mismo, 
se necesita estudiar todos estos factores que inciden en él. Para efectos teóricos este estudio describe algunas concepciones de investigadores que han estudiado los factores que incurren en el rendimiento académico y que además, son retomados en el estudio.

Olaz (2003, citado por Baquero et al, 2006) dice que:

Los intereses vocacionales son definidos como patrones de gustos, aversiones e indiferencias con respecto a actividades y ocupaciones relacionadas a una carrera. Asimismo, [...] el proceso de desarrollo vocacional incluye un modelo recíproco en el cual la autoeficacia y las expectativas de resultados influyen sobre los intereses. Es probable que las personas posean intereses perdurables en actividades en las cuales se consideren a sí mismos eficaces y en las cuales anticipen resultados positivos. Los intereses determinan a su vez las intenciones y metas que se fijará el sujeto, las cuales determinaran a su vez la elección de determinadas actividades y su práctica posterior (p. 42).

Hay que resaltar, que para llegar a formar estos hábitos es evidente que el ejercicio sea todos los días, considerando una hora uniforme. En la formación de hábitos es necesario el ejercicio; pero la práctica no conduce al aprendizaje. Saber si lo que hace está bien o mal, incita al individuo a modificar su conducta para que sea más eficiente en su estudio. Para llegar a formar los hábitos de estudio es necesario contar con tres factores principales como son psicológicos, ambientales e instrumentales.

En Robledo y García (2009) se describe que "las desventajas económicas y sociales tienen efectos adversos sobre el desarrollo cognitivo, socioemocional y escolar de los niños" (p.118), además agregan, "que los ingresos familiares pueden influir de manera indirecta en el bajo rendimiento de los alumnos debido a las escasas oportunidades de interacción con entornos estimulantes que tienen, a la limitación de recursos o a los conflictos derivados de esta escasez económica" (pp.118-119).

Zabalza (2011) menciona, que los métodos contienen cuatro dimensiones básicas como son la organización de los espacios y los tiempos, el modo de suministro de la información, la orientación y gestión de las actividades de aprendizaje y las relaciones interpersonales.

Hablar de metodología didáctica, además de métodos, es también tratar con estrategias didácticas. En el Modelo Educativo Normativa y Metodología para la Planificación Curricular de la Universidad Nacional Autónoma de Nicaragua, Managua (UNAN - Managua) se encuentran algunas estrategias didácticas que ayudan a los docentes en su práctica metodológica, bajo el paradigma de que ellas están centradas en el estudiante: Ubicación Contextual, Guías de cuestionamiento de lo que se aprende, Observación auto-reflexiva, Aprendizaje colaborativo, Estudios de caso, Aprendizaje por proyectos, Aprendizaje basado en la resolución de problemas, el Informe escrito analítico-reflexivo, Trabajo de campo, Conferencias magistrales (pp. 38-40). 


\section{MATERIALES Y MÉTODOS}

Por el método de investigación, el presente estudio es observacional, según el propósito del diseño metodológico el tipo de estudio es descriptivo (Piura, 2006). De acuerdo a la clasificación de Hernández, Fernández y Baptista (2006), el tipo de estudio es correlacional. De acuerdo al alcance de los resultados el estudio es analítico (Canales, Alvarado y Pineda, 1996).

A partir de los datos que fueron recolectados mediante la aplicación de una encuesta a todos los estudiantes del quinto año de la carrera de Banca y Finanzas activos dentro de la cohorte comprendida 2011-2015, equivalente a 39 estudiantes, se diseñó la base datos correspondiente, utilizando el software estadístico SPSS, v. 20 para Windows. Una vez que se realizó el control de calidad de los datos registrados, serán realizados los análisis estadísticos pertinentes.

Para Pedroza et al (2006), los métodos estadísticos utilizados para los análisis son:

\section{Medidas a Asociación para dos variables dicotómicas en tablas de contingencia.}

Coeficiente Phi: es una medida del grado de asociación entre dos variables dicotómicas, basadas en el estadístico de $\mathrm{Ji}$ - Cuadrado de Pearson, que toma valores entre 0 y 1 . Valores próximos a 0 , indicaran no asociación entre las variables y valores próximos a 1, indicarán una fuerte asociación. Al igual que Chi cuadrado, se construye a partir de las diferencias entre las frecuencias observadas y esperadas, solo que Phi toma valores entre 0 y 1 , en tablas $2 \times 2$ (p. 46).

\section{Medidas a Asociación para dos variables en escala nominal}

$V$ de Cramer: es otra extensión del Coeficiente Phi, ajustado al caso de que al menos una de las dos variables presente más de dos categorías, la cual a diferencia del Coeficiente de Contingencia, toma valores entre 0 y 1 , no dependiendo de una cota superior; sin embargo, la V de Cramer, tiende a subestimar el grado de asociación entre las variables. Valores de $\mathrm{V}$ de Cramer, próximos a 0 , indicarán no asociación entre las variables y valores próximos a 1, indicarán una fuerte asociación (p. 48).

\section{Medidas a Asociación para dos variables en escala ordinal}

Prueba de Tau -b de Kendall y Tau -c de Kendall: Tau -b de Kendall es una extensión de la prueba Gamma, en el sentido de que tanto la situación bajo al que puede se aplicada como su interpretación, es la misma. Sin embargo, presenta el inconveniente de que dichos valores solo pueden ser alcanzados cuando la tabla de contingencia sea cuadrada (2x2, 3x3, $4 \mathrm{x} 4$, etc.). 
Tau - c de Kendall es una corrección de la Tau -b de Kendall, para el caso de tablas de contingencia que contienen variables con distinto número de categorías. Frente a Tau -b, la prueba de Tau -c presenta la ventaja de poder alcanzar los valores de -1 y 1 , cuando el número de categorías de las dos variables es distinto. Sin embargo, tiene la desventaja de subestimar el verdadero grado de asociación entre las variables. Esta prueba es importante para analizar variables de tipo "Likert" (p. 51).

\section{RESULTADOS}

Los siguientes datos son los resultados de las distintas pruebas estadísticas aplicadas a diversas variables subjetivas y objetivas encontradas en la población en estudio, con dichas pruebas se permitió determinar la correlación o asociación existente entre ellas. En cada una de las correlaciones o asociaciones entre variables, se procede en plantear una hipótesis nula para contrastar su relación.

Se realizó el análisis de contingencia para las variables cuál es tu método de estudio independiente (visito la biblioteca) con procedencia de los estudiantes. El análisis de Cramer realizado, aportó las evidencias de $\boldsymbol{p}=\mathbf{0 , 3 2 4}$, que indica una respuesta estadística no significativa. Esto demuestra que los factores en estudio no presentan asociación entre sí, en este caso, significa que el método de estudio independiente de los estudiantes no está asociado por la procedencia de ellos.

\begin{tabular}{|c|c|c|c|}
\hline \multicolumn{2}{|l|}{} & Valor & Sig. aproximada \\
\hline Nominal por nominal & V de Cramer & .240 & .324 \\
\hline
\end{tabular}

Se realizó el análisis de contingencia para las variables cuál es tu método de estudio independiente (uso Internet) con procedencia de los estudiantes. El análisis de Cramer realizado, aportó las evidencias de $\boldsymbol{p}=\mathbf{0 , 1 7 3}$, que indica una respuesta estadística no significativa. Esto demuestra que los factores en estudio no presentan asociación entre sí, en este caso, significa que el método de estudio independiente de los estudiantes no está asociado por la procedencia de ellos.

\begin{tabular}{|c|c|c|c|}
\hline \multicolumn{2}{|l|}{} & Valor & Sig. aproximada \\
\hline Nominal por nominal & V de Cramer & .300 & .173 \\
\hline
\end{tabular}

Se realizó el análisis de contingencia para las variables cuál es tu método de estudio independiente (uso Internet) con promedio de ingreso familiar. El análisis de Cramer realizado, aportó las evidencias de $\boldsymbol{p}=\mathbf{0 , 7 4 4}$, que indica una respuesta estadística no significativa. Esto demuestra que los factores en estudio no presentan asociación entre sí, en 
este caso, significa que el método de estudio independiente de los estudiantes no está asociado al promedio familiar de sus hogares.

\begin{tabular}{|c|c|c|c|}
\hline \multicolumn{2}{|c|}{} & Valor & Sig. aproximada \\
\hline Nominal por nominal & V de Cramer & .194 & .744 \\
\hline
\end{tabular}

Se realizó el análisis de contingencia para las variables lugar donde accedes a Internet para estudio independiente (en un ciber) con promedio de ingreso familiar. El análisis de Cramer realizado, aportó las evidencias de $\boldsymbol{p}=\mathbf{0 , 8 4 3}$, que indica una respuesta estadística no significativa. Esto demuestra que los factores en estudio no presentan asociación entre sí, en este caso, significa que el lugar donde se accede a Internet para su estudio independiente no está asociado al promedio familiar.

\begin{tabular}{|c|c|c|c|}
\hline \multicolumn{2}{|l|}{} & Valor & Sig. aproximada \\
\hline Nominal por nominal & V de Cramer & .158 & .843 \\
\hline
\end{tabular}

Se realizó el análisis de contingencia para las variables frecuencia estudio de manera grupal con procedencia de los estudiantes. El análisis de Tau - C de Kendal realizado, aportó las evidencias de $\boldsymbol{p}=\mathbf{0 , 8 8 9}$, que indica una respuesta estadística no significativa. Esto demuestra que los factores en estudio no presentan correlación entre sí, en este caso, significa que la frecuencia estudio de manera grupal no está correlacionada con la procedencia de los estudiantes.

\begin{tabular}{|c|c|c|c|c|c|}
\hline \multicolumn{2}{|c|}{} & Valor & $\begin{array}{c}\text { Error típ. } \\
\text { asint. }^{\mathbf{a}}\end{array}$ & $\begin{array}{c}\text { T } \\
\text { aproximada }^{\text {b }}\end{array}$ & $\begin{array}{c}\text { Sig. } \\
\text { aproximada }^{2}\end{array}$ \\
\hline $\begin{array}{c}\text { Ordinal por } \\
\text { ordinal }\end{array}$ & $\begin{array}{c}\text { Tau-c de } \\
\text { Kendall }\end{array}$ & .016 & .113 & .140 & .889 \\
\hline
\end{tabular}

Se realizó el análisis de contingencia para las variables frecuencia de asistencia a clases con promedio de ingreso familiar. El análisis de Tau - C de Kendal realizado, aportó las evidencias de $\boldsymbol{p}=\mathbf{0 . 3 0 0}$, que indica una respuesta estadística no significativa. Esto demuestra que los factores en estudio no presentan correlación entre sí, en este caso, significa que la frecuencia de asistencia a clases no está correlacionada con promedio de ingreso familiar.

\begin{tabular}{|c|c|c|c|c|c|}
\hline \multicolumn{2}{|c|}{} & Valor & $\begin{array}{c}\text { Error típ. } \\
\text { asint. }^{\mathbf{a}}\end{array}$ & $\begin{array}{c}\text { T } \\
\text { aproximada }^{\mathbf{b}}\end{array}$ & $\begin{array}{c}\text { Sig. } \\
\text { aproximada }^{2}\end{array}$ \\
\hline $\begin{array}{c}\text { Ordinal por } \\
\text { ordinal }\end{array}$ & $\begin{array}{c}\text { Tau-c de } \\
\text { Kendall }\end{array}$ & -.081 & .078 & -1.036 & .300 \\
\hline
\end{tabular}


Se realizó el análisis de contingencia para las variables entrega de tareas en tiempo y forma con promedio de ingreso familiar. El análisis de Tau $-C$ de Kendal realizado, aportó las evidencias de $\boldsymbol{p}=\mathbf{0 , 4 0 1}$, que indica una respuesta estadística no significativa. Esto demuestra que los factores en estudio no presentan correlación entre sí, en este caso significa que la entrega de tareas en tiempo y forma no está correlacionada con el promedio de ingreso familiar.

\begin{tabular}{|c|c|c|c|c|c|}
\hline \multicolumn{2}{|c|}{} & Valor & $\begin{array}{c}\text { Error tip. } \\
\text { asint. }^{\mathbf{a}}\end{array}$ & $\begin{array}{c}\text { T } \\
\text { aproximada }^{\text {b }}\end{array}$ & $\begin{array}{c}\text { Sig. } \\
\text { aproximada }^{2}\end{array}$ \\
\hline $\begin{array}{c}\text { Ordinal por } \\
\text { ordinal }\end{array}$ & $\begin{array}{c}\text { Tau-c de } \\
\text { Kendall }\end{array}$ & .105 & .125 & .841 & .401 \\
\hline
\end{tabular}

Se realizó el análisis de contingencia para las variables frecuencia con la que usas Internet para estudio independiente con promedio de ingreso familiar. El análisis de Tau - C de Kendal realizado, aportó las evidencias de $\boldsymbol{p}=\mathbf{0 , 3 1 2}$, que indica una respuesta estadística no significativa. Esto demuestra que los factores en estudio no presentan correlación entre sí, en este caso significa que la frecuencia con la que se usa Internet para estudio independiente no está correlacionada con el promedio de ingreso familiar.

\begin{tabular}{|c|c|c|c|c|c|}
\hline \multicolumn{2}{|c|}{} & Valor & $\begin{array}{c}\text { Error tip. } \\
\text { asint. }^{\mathbf{a}}\end{array}$ & $\begin{array}{c}\text { T } \\
\text { aproximada }^{\text {b }}\end{array}$ & $\begin{array}{c}\text { Sig. } \\
\text { aproximada }^{2}\end{array}$ \\
\hline $\begin{array}{c}\text { Ordinal por } \\
\text { ordinal }\end{array}$ & $\begin{array}{c}\text { Tau-c de } \\
\text { Kendall }\end{array}$ & -.117 & .116 & -1.010 & .312 \\
\hline
\end{tabular}

Serealizó elanálisis de contingencia paralasvariablesfactor que desmotivaalestudiante en la carrera (la metodología y pedagogía de los docentes) con el tipo de material que usa el docente en el desarrollo de los contenidos de su asignatura (folletos). El análisis de Phi realizado, aportó las evidencias de $\boldsymbol{p}=\mathbf{0 . 6 3 2}$, que indica una respuesta estadística no significativa. Esto demuestra que los factores en estudio no presentan asociación entre sí, en este caso, significa que el factor que desmotiva al estudiante en la carrera no está asociado al tipo de material que usa el docente en el desarrollo de los contenidos de su asignatura.

\begin{tabular}{|c|c|c|c|}
\hline \multicolumn{2}{|l|}{} & Valor & Sig. aproximada \\
\hline Nominal por nominal & Phi & .077 & .632 \\
\hline
\end{tabular}

Se realizó el análisis de contingencia para las variables factor que desmotiva al estudiante en la carrera (la metodología y pedagogía de los docentes) con el tipo de material que usa el docente en el desarrollo de los contenidos de su asignatura (libro de texto). El análisis de Phi realizado, aportó las evidencias de $\boldsymbol{p}=\mathbf{0 . 3 3 0}$, que indica 
una respuesta estadística no significativa. Esto demuestra que los factores en estudio no presentan asociación entre sí, en este caso, significa que el factor que desmotiva al estudiante en la carrera no está asociado al tipo de material que usa el docente en el desarrollo de los contenidos de su asignatura.

\begin{tabular}{|c|c|c|c|}
\hline \multicolumn{2}{|l|}{} & Valor & Sig. aproximada \\
\hline Nominal por nominal & Phi & .156 & .330 \\
\hline
\end{tabular}

Se realizó el análisis de contingencia para las variables aspecto personal que te motivó a estudiar tu carrera con conoces el pénsum de tu carrera. El análisis de Phi realizado, aportó las evidencias de $\boldsymbol{p}=\mathbf{0 . 5 2 5}$, que indica una respuesta estadística no significativa. Esto demuestra que los factores en estudio no presentan asociación entre sí, en este caso, significa que el aspecto personal que le motivó a estudiar su carrera no está asociado al factor conocer el pénsum de su carrera.

\begin{tabular}{|c|c|c|c|}
\hline \multicolumn{2}{|l|}{} & Valor & Sig. aproximada \\
\hline Nominal por nominal & Phi & -.102 & .525 \\
\hline
\end{tabular}

Se realizó el análisis de contingencia para las variables aspecto personal que te motivó a estudiar tu carrera con conoces el perfil profesional de su carrera. El análisis de Phi realizado, aportó las evidencias de $\boldsymbol{p}=\mathbf{0 . 5 2 5}$, que indica una respuesta estadística no significativa. Esto demuestra que los factores en estudio no presentan asociación entre sí, en este caso, significa que el aspecto personal que le motivó a estudiar la carrera no está asociado al factor conocer el perfil profesional de su carrera.

\begin{tabular}{|c|c|c|c|}
\hline \multicolumn{2}{|l|}{} & Valor & Sig. aproximada \\
\hline Nominal por nominal & Phi & -.102 & .525 \\
\hline
\end{tabular}

Se realizó el análisis de contingencia para las variables factor que desmotiva al estudiante en la carrera con forma en que se desarrollaba la clase de Metodología de la Investigación. El análisis de Cramer realizado, aportó las evidencias de $\boldsymbol{p}=\mathbf{0 , 5 0 4}$, que indica una respuesta estadística no significativa. Esto demuestra que los factores en estudio no presentan asociación entre sí, en este caso, significa que el factor que desmotiva al estudiante en la carrera no está asociado con la forma en que se desarrollaba la clase de Metodología de la Investigación.

\begin{tabular}{|c|c|c|c|}
\hline \multicolumn{2}{|l|}{} & Valor & Sig. aproximada \\
\hline Nominal por nominal & V de Cramer & .210 & .504 \\
\hline
\end{tabular}


Se realizó el análisis de contingencia para las variables factor que desmotiva al estudiante en la carrera con forma en que se desarrollaba la clase de Investigación Aplicada. El análisis de Cramer realizado, aportó las evidencias de $\boldsymbol{p}=\mathbf{0 , 0 4 5}$, que indica una respuesta estadística significativa. Esto demuestra, que los factores en estudio presentan asociación entre sí, en este caso, significa que el factor que desmotiva al estudiante en la carrera está asociado con la forma en que se desarrolló la clase de Investigación Aplicada.

\begin{tabular}{|c|c|c|c|}
\hline \multicolumn{2}{|l|}{} & Valor & Sig. aproximada \\
\hline Nominal por nominal & V de Cramer & .409 & .045 \\
\hline
\end{tabular}

Se realizó el análisis de contingencia para las variables uso de las TIC para el desarrollo de los contenidos de la asignatura Metodología de la Investigación con el tipo de material que usa el docente en el desarrollo de los contenidos. El análisis de Cramer realizado, aportó las evidencias de $\boldsymbol{p}=\mathbf{0 , 5 4 5}$, que indica una respuesta estadística no significativa. Esto demuestra que los factores en estudio no presentan asociación entre sí, en este caso, significa que el uso de las TIC para el desarrollo de los contenidos de la asignatura Metodología de la Investigación no está asociado con el tipo de material que usa el docente en el desarrollo de los contenidos.

\begin{tabular}{|c|c|c|c|}
\hline \multicolumn{2}{|l|}{} & Valor & Sig. aproximada \\
\hline Nominal por nominal & V de Cramer & .285 & .545 \\
\hline
\end{tabular}

Se realizó el análisis de contingencia para las variables uso de las TIC para el desarrollo de los contenidos de la asignatura Investigación Aplicada con el tipo de material que usa el docente en el desarrollo de los contenidos. El análisis de Cramer realizado, aportó las evidencias de $\boldsymbol{p}=\mathbf{0 , 1 9 0}$, que indica una respuesta estadística no significativa. Esto demuestra que los factores en estudio no presentan asociación entre sí, en este caso, significa que el uso de las TIC para el desarrollo de los contenidos de la asignatura Investigación Aplicada no está asociado con el tipo de material que usa el docente en el desarrollo de los contenidos.

\begin{tabular}{|c|c|c|c|}
\hline \multicolumn{2}{|l|}{} & Valor & Sig. aproximada \\
\hline Nominal por nominal & V de Cramer & .407 & .190 \\
\hline
\end{tabular}

Se realizó el análisis de contingencia para las variables conoce el perfil profesional de su carrera con factor que te determinó a elegir tu carrera. El análisis de Cramer realizado, aportó las evidencias de $\boldsymbol{p}=\mathbf{0 , 0 0 0}$, que indica una respuesta estadística altamente significativa. Esto demuestra que los factores en estudio presentan asociación entre sí, en este caso, significa que el conocer el perfil profesional de su carrera está asociado con el factor, determinó a elegir su carrera. 


\begin{tabular}{|c|c|c|c|}
\hline \multicolumn{2}{|l|}{} & Valor & Sig. aproximada \\
\hline Nominal por nominal & V de Cramer & 1.000 & .000 \\
\hline
\end{tabular}

\section{DISCUSIÓN DE RESULTADOS}

En su libro "Sistema de Análisis Estadísticos con SPSS", Pedroza et al (2006), facilita un indicador para determinar si existe la asociación, relación o correlación entre los factores analizados. Pedroza refiere que realizando la comparación de los resultados de las pruebas estadísticas con el nivel de significación establecido (para el estudio $\alpha=0.05$ ) se dará respuesta a la hipótesis planteada por el investigador. En el apartado de resultados estadí sticos se muestran los datos obtenidos de la aplicación de las pruebas estadísticas en la que se comprueba, que en el estudio no da lugar de asociación, relación o correlación de algunos factores analizados.

Se presenta las hipótesis sobre la asociación, relación o correlación de los factores:

Ho: El factor que desmotiva al estudiante en la carrera (la metodología y pedagogía de los docentes) no está asociado al tipo de material que usa el docente durante el desarrollo de los contenidos (folletos).

Ho: El factor que desmotiva al estudiante en la carrera (la metodología y pedagogía de los docentes) no está asociado al tipo de material que usa el docente durante el desarrollo de los contenidos (libros de textos).

La literatura hace hincapié en que la metodología y pedagogía, utilizada por los docentes en los encuentros de clase, es un elemento que influye directamente proporcional en la motivación que tendrán los estudiantes hacia esa materia que se esté impartiendo. En el estudio se comparó la existencia de asociación entre la metodología y pedagogía que tienen los docentes al hacer uso del tipo de material didáctico para impartir sus disciplinas académicas y la motivación de los estudiantes en el aprendizaje de esa disciplina, los datos estadísticos de las pruebas revelan, que en el estudio no se cumple tal asociación ( $\boldsymbol{p}=\mathbf{0 . 6 3 2}$ y $\boldsymbol{p}=\mathbf{0 . 3 3 0}$ respectivamente mayores al $\alpha=0.05$ ), es decir, para los estudiantes, el material que utilice el docente no es el factor que esté provocando la desmotivación en el estudiante al estudiar la carrera.

Ho: La forma en que se desarrollaba la clase de la Asignatura Metodología de la Investigación no será el factor que desmotive al estudiante en la carrera (la metodología y pedagogía de los docentes).

Ho: La forma en que se desarrollaba la clase de la Asignatura Investigación Aplicada no será el factor que desmotive al estudiante en la carrera (la metodología y pedagogía de los docentes).

Otro elemento que se presenta en la motivación del estudiante en el proceso enseñanzaaprendizaje es el ambiente que crea el docente en el desarrollo de la clase presencial, se cree que este elemento tiene influencia directa. En el estudio, se procedió en analizar el grado de 
asociación entre estos factores, utilizando como parámetro, las asignaturas de Metodología de la Investigación e Investigación Aplicada, los resultados de las pruebas estadísticas demuestran que: en Metodología de la Investigación, el resultado $\boldsymbol{p}=\mathbf{0 . 5 0 4}$ (mayor al $\alpha=0.05$ ) determina que no existe asociación, lo que indica la presencia de otros factores causantes de la desmotivación como los que se mencionan en el apartado teórico en pedagogía y metodología de enseñanza.

En Investigación Aplicada, el escenario tiene otro sentido. El dato que arroja la prueba $\boldsymbol{p}=\mathbf{0 . 0 4 5}$ (menor al $\alpha=0.05$ ) indica, que la forma en que se desarrolló la clase presencial sí tuvo influencia sobre la desmotivación del estudiantado. Hay que destacar, que el estudiante al cursar esta asignatura posee experiencia sobre cómo evaluar el desempeño metodológico y pedagógico del docente puesto que ha cursado 8 semestres.

Ho: El uso de las TIC para el desarrollo de los contenidos de la Asignatura Metodología de la Investigación no influirá en el tipo de material que use el docente en el desarrollo de los contenidos (folleto).

Ho: El uso de las TIC para el desarrollo de los contenidos de la Asignatura Investigación Aplicada no influirá en el tipo de material que use el docente en el desarrollo de los contenidos (libros de textos).

En cuanto a las herramientas tecnológicas que utiliza el docente en su gestión académica, tanto en la asignatura Metodología de la Investigación e Investigación Aplicada, se demuestra que no existe asociación con el tipo de material que usa $(\boldsymbol{p}=\mathbf{0 . 5 4 5}$ y $\boldsymbol{p}=\mathbf{0 . 1 9 0}$ respectivamente mayores al $\alpha=0.05)$. Esto se debe a que los docentes de estas asignaturas emplean enfoques tradicionalistas en su enseñanza, indicando que, los métodos y estrategias son tradicionales "folletos o libros de textos". Una de las manifestaciones del uso de las herramientas tecnológicas en los docentes es simplemente para obtener la información y realizar una réplica del material digital, aun sabiendo de la gran cobertura de conocimiento que puede explotar para luego ser compartido con sus estudiantes.

Ho: El factor aspecto personal que le motivó a estudiar su carrera (Me gustaba) no está asociado a Conocer el pénsum de su carrera.

Ho: El factor aspecto personal que le motivó a estudiar su carrera (Me gustaba) no está asociado a Conocer el perfil profesional de su carrera.

Cuando se desea estudiar una carrera de profesionalización, diversidad de opiniones convergen en que se debe tener una vocación ante tal decisión y muchos lo familiarizan con los gustos y preferencias que tiene el individuo, situación que lo motiva a efectuar la acción. Al realizar las pruebas estadísticas para comprobar la existencia de asociación entre los factores plasmados en las hipótesis, se revela que los factores Conoces el pénsum y perfil profesional de tu carrera, no son causa que determinen que los estudiantes se motivaron en estudiar la carrera 
de Banca y Finanzas (ambos con un $\boldsymbol{p}=\mathbf{0 . 5 2 5}$ mayores al $\alpha=0.05$ ), sino que ellos eligieron esta carrera por otros parámetros.

Ho: Conocer el perfil profesional de la carrera no influirá en determinar la elección de la carrera.

Cuando se realizó la prueba estadística para conocer la asociación entre los factores mencionados en la hipótesis, se obtuvo la existencia de tal asociación, demostrando que el estudiante sabía a lo que se iba a enfrentar en el campo laboral una vez egresado.

Ho: La procedencia de los estudiantes no interviene en la selección del método de estudio independiente (visito la biblioteca).

Ho: La procedencia de los estudiantes no interviene en la selección del método de estudio independiente (uso Internet).

Ho: La frecuencia de estudio de manera grupal no está asociada a la procedencia del estudiante.

En el área empírica, se cree que el rendimiento académico es influenciado en un alto grado por la procedencia geográfica de los estudiantes, destacando que aquellos que provienen del sector rural presentan dificultades en el desarrollo de su vida estudiantil puesto que no cuentan con las herramientas necesarias para enfrentar las demandas académicas; la distancia y los recursos económicos les impide mantener constantes encuentros en los grupos de estudio entre otras hipótesis. Los datos obtenidos en la encuesta demuestran que aproximadamente el 23 \% de la población estudiantil de la cohorte de la carrera Banca y Finanzas proviene de la zona rural.

Al aplicar las pruebas estadísticas para determinar el grado de asociación y correlación entre los factores citados en las hipótesis, se obtuvo como resultado que no hay asociación entre la procedencia con el método de estudio independiente, sea por uso de Internet o visitar la biblioteca, situación que dependió de las características que se encontró en el grupo de estudio, pues existe gran influencia por contar con más de la mitad de la población proveniente de la zona urbana, al comparar con la frecuencia de estudio grupal, de igual manera, no se evidencia la correlación.

Este hallazgo es complementado con otros resultados de pruebas realizadas en las que se evidencia que tienen acceso a usar los medios tecnológicos y la sociabilidad de compartir conocimiento entre compañeros para su estudio independiente sin muchos obstáculos.

Ho: El promedio de ingreso familiar en los hogares de los estudiantes no interviene en la selección del método de estudio independiente (uso Internet).

Ho: El promedio de ingreso familiar en los hogares de los estudiantes no será un factor que interviene con el lugar en donde accede a Internet para su estudio independiente (en un ciber). 
Los diversos investigadores han establecido en sus artículos, que el rendimiento académico está ligado con el factor “ingreso económico que dispone el individuo", considerándose como uno de los principales elementos que favorece o desfavorece al estudiante. En el estudio se procedió en realizar correlación para verificar el grado de asociación entre los factores descritos en las hipótesis, los datos empíricos arrojan un $\boldsymbol{p}=\mathbf{0 . 7 4 4}$ y $\boldsymbol{p}=\mathbf{0 . 8 4 3}$ respectivamente (mayores al $\alpha=0.05$ ) demostrando que: el promedio de ingreso que poseen los estudiantes en la cohorte en estudio no fue obstáculo para que ellos seleccionaran el método de estudio que se ajustará a la satisfacción de sus necesidades. En el mundo moderno que se vive, la tecnología facilita obtener los conocimientos científicos de cualquier área de la ciencia de forma accesible y con mayor variedad de fuentes.

Aun cuando el estudiante no posee los recursos económicos suficientes para hacerle frente a los costos de estudiar bajo la vía tecnológica (el uso de Internet requiere disponer de una computadora y el contrato de servicios) no le impide acceder a ella, pues hace uso de los servicios de cibercafé existentes en la zona donde vive o estudia, pero ¿qué impacto tiene su promedio de ingreso familiar para poder acceder a ella? El resultado de la prueba estadística afirma que no es un factor que restrinja al estudiante debido a que el costo de acceso es favorable.

Ho: La frecuencia de asistencia a clases no está asociada al promedio de ingresos que perciben los hogares de los estudiantes.

Ho: La entrega de tareas en tiempo y forma no está asociada al promedio de ingresos que perciben los hogares de los estudiantes.

Ho: La frecuencia con la que usa Internet para estudio independiente no está asociada al promedio de ingresos que perciben los hogares de los estudiantes.

De igual manera, se procedió a realizar pruebas de correlación para los factores descritos en las hipótesis para determinar si el promedio de ingreso de las familias influye en ellos. En las tres hipótesis el resultado es que no hay correlación puesto que sus $\boldsymbol{p}$-valores resultaron mayores a $\alpha=0.05$, lo que continúa afirmando que en los estudiantes de la cohorte 2011-2015 no le ha sido obstáculo el disponer de recursos económicos limitados para obtener una buena preparación académica.

\section{CONCLUSIÓN}

Utilizando los factores subjetivos y objetivos identificados con mayor incidencia en el rendimiento académico de la carrera descritos en el artículo factores subjetivos y objetivos incidentes en el rendimiento académico en la carrera Banca y Finanzas, FAREM-Carazo, UNAN-Managua y mediante la aplicación de las pruebas estadísticas no paramétricas $\mathrm{V}$ de Cramer, Tau - C de Kendall y Phi se determinó que un 88 \% de las correlaciones no presentan 
asociación entre los factores subjetivos y objetivos que inciden en el rendimiento académico de los estudiantes de la carrera de Banca y Finanzas de la FAREM-Carazo, UNAN-Managua, cohorte 2011-2015; comprobando que las hipótesis que se plantean las autoridades, la comunidad universitaria y la sociedad respecto a tales factores no se cumplen de forma generalizada, sino que depende de las características particulares de la población a estudiar.

\section{BIBLIOGRAFÍA}

Baquero C., Menjura E. y Tobón M.. (2006). Motivación, autoeficacia y plan de carrera en estudiantes de Psicología de la Universidad Cooperativa de Colombia, seccional Bogotá. Revista de la Facultad de Psicología Universidad Cooperativa de Colombia, 3(3), 39-48.

Canales, Alvarado y Pineda. (1996). Metodología de la Investigación, Manual para el Desarrollo de personal de Salud. Oficina Regional de la Organización Mundial de la Salud, OPS (2da ed.). Washington, D.C. 20037, E.U.A.

Hernández, Fernández y Baptista. (2006). Metodología de la Investigación (5ta ed.). México: Mc Graw-Hill.

Pedroza, H. y Dicovskei, L. (2006). Sistema de Análisis Estadísticos con SPSS. Managua: Instituto Nicaragüense de Tecnología Agropecuaria.
Pedroza, M. (2016). Uso de los Modelos Univariados, Regresión y Correlación, en estudios de Tipo Observacional. Managua: UNAN - Managua.

Piura, J. (2006). Metodología de la Investigación Científica: Un Enfoque Integrador (1ra ed.). Managua: PAVSA.

Robledo, P. y García, J. (2009). El entorno familiar y su influencia en el rendimiento académico de los alumnos con dificultades de aprendizaje: revisión de estudios empíricos. Aula Abierta, 37(1), 117-128.

UNAN - Managua. (2011). Modelo Educativo, Normativa y Metodología para la Planificación Curricular 2011. Managua: UNAN - Mangua.

Zabalza, M. (2011). Metodología docente. Revista de Docencia Universitaria, 9(3), 75-98. 